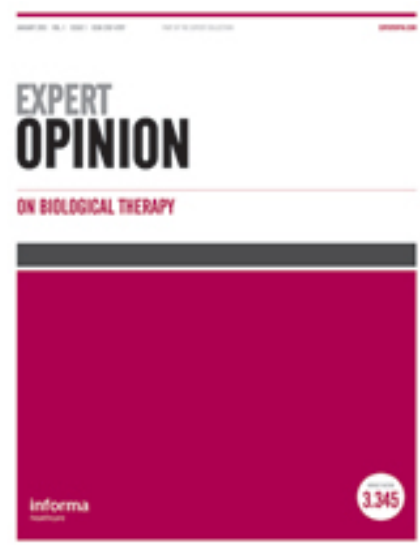

Please download and read the instructions before proceeding to the peer review

Ocrelizumab not recommended in France for patients with primary progressive multiple sclerosis while recommended in England: a review comparing the assessment by HAS and NICE

\begin{tabular}{|r|l|}
\hline Journal: & Expert Opinion On Biological Therapy \\
\hline Manuscript ID & EOBT-2020-ST-0223.R1 \\
\hline Manuscript Type: & Review \\
\hline Keywords: & $\begin{array}{l}\text { primary progressive multiple sclerosis, Ocrelizumab, NICE, HAS, Health } \\
\text { technology assessment, England, France }\end{array}$ \\
\hline \multicolumn{2}{|l}{} \\
\hline
\end{tabular}

\title{
SCHOLARONE ${ }^{m}$ Manuscripts
}




\title{
Ocrelizumab not recommended in France for patients with primary progressive multiple
} sclerosis while recommended in England: a review comparing the assessment by HAS and NICE

Xavier Armoiry ${ }^{1,2}$, Hans-Martin Späth ${ }^{3}$, Anna-Maria Henaine ${ }^{4}$, Claude Dussart ${ }^{3}$, Carl Counsel1 ${ }^{5}$, Martin Connock ${ }^{2}$

${ }^{1}$ University of Lyon, School of Pharmacy (ISPB) / UMR CNRS 5510 MATEIS / Lyon University Hospitals, “Edouard Herriot” Hospital, Pharmacy Department, Lyon, France

${ }^{2}$ University of Warwick, Warwick Medical School, Gibbet Hill Road, CV47AL Coventry, UK

${ }^{3}$ University of Lyon, University Lyon 1, EA 4129 Parcours Santé Systémique, Lyon France.

${ }^{4}$ Lebanese University, School of Pharmacy, Lebanon.

${ }^{5}$ University of Aberdeen, Institute of Applied Health Sciences, AB25 2ZD, Aberdeen, UK.

\author{
Author for correspondence: \\ Prof. Xavier Armoiry, PharmD, PhD. University of Lyon, Claude Bernard University Lyon 1 \\ School of Pharmacy (ISPB), Public Health department, 8 avenue Rockefeller, 69008 Lyon, \\ France, email: xavier.armoiry@univ-lyon1.fr , Tel: +33 (0)472 110854
}

\begin{abstract}
Introduction: Ocrelizumab is the first approved drug for primary progressive multiple sclerosis. Following appraisal by health technology assessment (HTA) bodies, this medicine
\end{abstract}


has not been widely covered across European countries. We have compared the HTA process in England and France.

Area covered: We undertook an analysis of relevant documents that were published by the two HTA bodies. We analysed patients' availability of ocrelizumab at the different stages of the process.

Expert opinion: We identified differences in the assessment, one being the use of a different population of the pivotal trial, which has resulted in the consideration of distinct clinical effectiveness estimates. Ocrelizumab became available earlier in France as part of an early access programme. However, rapid access was discontinued for newly eligible patients following an opinion concluding that Ocrelizumab yielded no additional benefit over placebo. This opinion was not compatible with criteria allowing reimbursement in France.

In England, there was no early access programme and following an appraisal that included cost-effectiveness evaluation combined with pricing agreements, the medicine was finally recommended. In conclusion, differences in the HTA process may result in appreciable differences in timing and outcome from marketing authorisation to adoption of newly licensed drugs.

Keywords: NICE, HAS, Health technology assessment, France, England, Ocrelizumab, primary progressive multiple sclerosis

\section{ARTICLE HIGHLIGHTS}

- Ocrelizumab is the first approved drug to treat patients with primary progressive multiple sclerosis (PPMS). In Europe, this medicine has not been widely covered across countries.

- We have compared the HTA process in England and France which has resulted in different outcomes in these two countries. 
- Our comparison identified differences in the paradigm of assessment, as well as on the evidence submitted by the Company.

- Differences in the HTA process may result in appreciable differences in timing and outcome from marketing authorisation to health service adoption of newly licensed drugs. 


\section{Introduction}

Ocrelizumab is a monoclonal antibody that selectively binds to and depletes CD20-expressing B cells. It has been developed to treat patients with either relapsing remitting multiple sclerosis (RRMS) or with primary progressive multiple sclerosis (PPMS).

The evaluation of ocrelizumab in both forms of multiple sclerosis (MS) has been subject to separate clinical development by the manufacturer Roche which has led to two label indications granted by the European Medicine Agency (EMA) on the basis of distinct pivotal clinical trials $[1,2]$. These approvals were obtained simultaneously for RRMS and PPMS in January 2018 [3].

Subsequently, ocrelizumab treatment in each indication has been appraised by countryspecific health technology assessment (HTA) bodies for the purpose of decision making for reimbursement within National Health Insurance Systems (NHIS).

The context of assessment was notably different according to the two label indications. Indeed, RRMS is a type of MS for which a great number of approved therapeutic options are available [4], ranging from conventional beta-interferons and glatiramer acetate [5], orally taken disease modifying therapies such as teriflunomide [6], to monoclonal antibodies to treat highly active forms [7]. Conversely, before the marketing approval of ocrelizumab, there was no licensed treatment for patients with PPMS [8] although off-label use of agents such as antiCD20 rituximab has been described [9]. 
Since the granting of marketing authorisation for ocrelizumab in PPMS, a number of countries in Europe such as Germany, Switzerland, Scotland, and England have provided a positive opinion on its reimbursement [10-16].

As of September 2020, France is one of the countries in this zone that has decided not to support the reimbursement of ocrelizumab in PPMS by the NHIS while the RRMS indication has received positive coverage [17].

In this paper, our objective was to compare the HTA process in France and England and to assess their differences and limitations using the example of ocrelizumab for PPMS as a case study.

Here, we define the HTA process in terms of the main hurdles between the start of appraisal until the final decision on reimbursement.

Our focus of interest is the comparison of French and English HTA processes because of the central functional roles played by the National Authority for Health (HAS) in France and the National Institute for Health and Care Excellence (NICE) in England in the assessment of health technologies and in ensuring rational price settings. 


\section{Body}

We briefly describe the overall processes for drug assessment in France and England, then we present the methods for our case-study comparing HTA and the decision-making processes for ocrelizumab in both countries.

\section{Overview of national drug assessment in France and England}

Briefly, in England, NICE determine whether a newly approved drug is effective and costeffective for patients treated within the NHS in England [18,19].

Cost-effectiveness is judged against the threshold of $£ 30,000$ per Quality Adjusted Life Year (QALY) gained compared to relevant comparator(s) [20]. Only new medicines which have been deemed as likely to incur significant increased expenditures for the National Health Service (NHS) are subject to appraisals by NICE [21].

In the event cost-effectiveness is not achievable with the drug list price, companies are invited to propose commercial access agreements that are taken into account in appraising the drug.

In France, the formal assessment process starts with consideration by the Transparency Committee of the HAS so as to determine the level of actual medical benefit (SMR for "Service Médical Rendu") on the basis of criteria such as the clinical effectiveness and safety profile. Then, if SMR is judged sufficient (i.e. SMR is weak, moderate or important) rather than insufficient, the level of improvement of medical benefit (ASMR for “Amélioration du Service Médical Rendu") is assessed looking for added clinical benefit of the new drug relative to existing treatment(s). ASMR ratings range from I (major benefit) to $\mathrm{V}$ (absence of/no additional benefit) [22].

To ensure greater clarity on decisions made by the Transparency Committee, the principle of judgement to determine SMR and ASMR ratings have recently been published [23]. 
Based on the ASMR rating, together with consideration of other factors such as the size of the target population, and price of comparator, the following step is the pricing discussion between the National Economic Committee (CEPS for "Comité Economique des Produits de Santé") and the drug Company [24].

Prior to the pricing discussion, an important additional stage applies specifically for drugs for which administration requires an inpatient hospital stay (as is essentially the case for intravenously administered agents such as ocrelizumab). This stage involves assessing whether the drug is eligible for registration on the list of health technologies refundable beyond hospital stay tariffs; in France this is better known as the "T2A list". Formal criteria operate to determine T2A list eligibility [25]; among these a drug must have "major" or "important" SMR rating and most crucially must have obtained ASMR rating of I to III, or possibly IV but only if the health condition is judged to present some "public health interest", which is assessed based on published criteria [23]. Outside of these circumstances, registration on T2A list for drugs with ASMR ratings of IV or $\mathrm{V}$ is possible only if the relevant comparator of the drug, as assessed by HAS, is itself already registered on the $T 2 A$ list [25].

Should a drug be granted $T 2 A$ list registration, pricing discussion will occur as previously described whereas it will not in case $T 2 A$ list registration is not possible. In case drugs are funded through DRGs'tariffs, pricing discussion takes place directly between companies and hospitals.

Last, it is worth noting that unlike NICE, HAS restricts examination of the cost-effectiveness of new technologies to those deemed innovative (defined as therapies, whether drugs or medical devices, for which manufacturers claim ASMR rating of I to III) and if the anticipated national yearly budget is at least 20 million euros)[26]. In the case of ocrelizumab, the drug met the two 
criteria requiring a cost-effectiveness evaluation to be assessed by the Committee of Economic Evaluation and Public Health (CEESP) of the French HAS [27]. Cost-effectiveness opinions by CEESP are intended to be taken into account by the CEPS at the stage of pricing, should pricing take place, despite no defined threshold to judge whether a new technology is costeffective [28].

\begin{abstract}
Ahead of the formal assessment process previously described, there is in France a possibility for an early access scheme called ATU (“Autorisation Temporaire d'Utilisation”). ATUs are granted by the French regulatory agency of medicines (ANSM) before formal EMA approval for some drugs. The principle of ATU scheme, which enables drug-related expenses to be fully covered by the NHIS, is to allow access for patients essentially where the product is expected to provide clinical benefit in the absence of alternatives [29].
\end{abstract}

\title{
Methods of our case-study
}

In the work, we used observational methods [30].

The HTA processes related to ocrelizumab were examined and compared using all relevant documents within the internet websites of HAS and NICE.

For NICE, we identified the following documents [12]: draft and final scope, committee papers, appraisal consultation document (ACD), final appraisal document (FAD), guidance.

For HAS, we identified the transparency committee opinion, the minutes of transparency committee meetings, and the opinion by the CEESP [10]. 


\section{Description of our case-study}

Figure 1 depicts the main milestones of the assessment of ocrelizumab in England and France and shows the resulting decisions regarding availability of the drug for newly eligible patients.

Eleven months following the publication of the pivotal phase III trial [1], and one month before ocrelizumab was granted a marketing authorisation [3], France was the first country allowing access to ocrelizumab for patients with PPMS. Early use of ocrelizumab was possible through the ATU access scheme. From early December 2017 to late February 2018, 56 patients benefited from ocrelizumab within this scope [31]. It was restricted to people with early PPMS in terms of disease duration and level of disability, and with imaging features characteristic of inflammatory activity.

From 21 February 2018 onwards, ocrelizumab became available in France only for patients who were already initiated on treatment, this meant that ocrelizumab for newly eligible patients was no longer possible. It is not clear from publicly available documents why discontinuation to access for newly eligible patients was decided, but this may be related to the concomitant appraisal of ocrelizumab by HAS.

The first appraisal committee meetings at HAS and at NICE took place in April 2018 and June 2018, respectively.

Both HTA bodies have assessed the clinical effectiveness of ocrelizumab according to its label indication, namely people with early PPMS in terms of disease duration and level of disability, and with imaging features characteristic of inflammatory activity.

Regarding the scope of the appraisal, both NICE and HAS considered that there was no relevant active comparator for ocrelizumab in PPMS. However, based on reported off-label use of 
rituximab, HAS regretted the absence of direct comparison between ocrelizumab and rituximab in the submission by the Company [10].

The main source of evidence submitted by the manufacturer of ocrelizumab (Roche) to support the clinical effectiveness relied on the findings from the ORATORIO trial [1].

ORATORIO is a randomised controlled trial (RCT) that enrolled 732 PPMS patients who received in a 2:1 ratio either ocrelizumab $(n=488)$ or placebo $(n=244)$. The primary endpoint was the proportion of patients with disability progression (CDP) confirmed for 12 weeks (CDP12). Secondary endpoints were CDP confirmed for 24 weeks (CDP-24), time of 25-foot walk test $(\mathrm{T} 25 \mathrm{FW})$, change in $\mathrm{T} 2$ brain lesions on MRI, percentage change in total brain volume, and change in physical component summary score of the SF-36 [16].

Neither NICE nor HAS have identified major methodological issues with the design or the conduct of the ORATORIO trial which was judged to be at low risk of bias $[10,12]$.

Unlike the Transparency Committee at HAS, NICE pointed out that CDP-24 had more clinical relevance for a disease like PPMS than did CDP-12 [10].

Based on results for the intention to treat (ITT) population, both HAS and NICE have acknowledged the demonstration of superiority of ocrelizumab over placebo but only HAS qualified this by indicating the gain in delay to confirmed disability was modest and of unclear clinical relevance.

HAS rationale was founded on the small absolute benefit $(<4 \%)$ in the proportion of people with disability progression confirmed for 12 weeks following 120 weeks on treatment $(30.2 \%$ in the ocrelizumab arm vs $34.0 \%$ in the placebo group). However, at the time of statistical analyses pre-planned by ORATORIO's investigators that included all patients with a minimum of 120 weeks on treatment (i.e. most of patients had more than 120 weeks on treatment), the 
absolute benefit was a little greater $(+6.4 \%: 32.9 \%$ in the ocrelizumab arm vs $39.3 \%$ in the placebo arm).

The absence of clear improvement on health-related quality of life was emphasized $[10,12]$. Unlike NICE, HAS have put greater emphasis on uncertainties regarding the potential for ocrelizumab-induced lymphodepletion in such a long-term condition as PPMS [10].

A notable difference in the appraisal by NICE and HAS pertains to the nature of submitted results from the ORATORIO trial.

At HAS level, the results presented by Roche seemed to be only based on the ITT population of this trial, which appears to only partially match to the label indication of ocrelizumab (early PPMS in terms of disease duration and level of disability, and with imaging features characteristic of inflammatory activity) since $\approx 75 \%$ of included patients had no T1 gadolinium (Gd) enhancing lesions and $\approx 11 \%$ had 0 to 5 T2 Gd enhancing lesions [10].

Conversely, at NICE level, Roche submitted not only the results from the ORATORIO ITT population but also those from a post-hoc defined magnetic resonance imaging (MRI) active subgroup, matching the EMA label indication. MRI disease activity was defined according to the presence of $\mathrm{T} 1 \mathrm{Gd}-$ enhancing or new $\mathrm{T} 2$ lesions between screening and baseline, and was deemed by the Company to be a good match to the marketing authorisation of 'early and active' disease [16].

The analyses submitted within this scope had the advantage of reporting results from a subset of the ORATORIO population matching most closely to the label indication, which may derive greater benefit from ocrelizumab. However, these data suffer the inherent disadvantages of a post-hoc analysis especially since the sample size was more than halved from 732 to 293 
patients ( $\mathrm{n}=189$ in the ocrelizumab arm; $\mathrm{n}=104$ in the placebo arm) [16]. This small number of patients, on which all the clinical effectiveness evidence was based, contrasts with the high number of patients who are likely to be treated worldwide by the drug.

Presumably in order to consider the population with the greatest match to the label indication, HAS also examined the results of ORATORIO in patients with T1Gd lesions at inclusion, which was a pre-specified subgroup analysis, and noted the absence of difference between ocrelizumab and placebo (CDP-12 HR 0.65, 95\%CI 0.4-1.06). While the test of interaction on this subgroup analysis was negative $(\mathrm{p}=0.2076)$ it lacked power since the $\mathrm{T} 1 \mathrm{Gd}+$ subgroup only comprised only 193 patients in total (133 in the ocrelizumab arm/60 in the placebo arm).

In England, despite the post-hoc nature of the analysis, NICE concluded that it was appropriate for decision making on clinical effectiveness to use data from the 'MRI-active' subgroup rather than the ITT population of ORATORIO. This opinion was based in part on clinical experts' opinions who agreed on the proposed criteria to define MRI activity in patients with PPMS.

Using data from the post-hoc defined MRI-active subgroup and considering the primary endpoint, the magnitude of benefit of ocrelizumab over placebo appeared slightly greater than in the ITT population (CDP-12 HR 0.68 [95\%CI 0.46- 0.99] vs 0.76 [95\%CI 0.59- 0.98] respectively). Using the same source of data and considering the most relevant assessment of confirmed disability progression, namely CDP-24, a similar trend was observed (CDP-24 HR 0.71 [95\%CI 0.47- 1.06] vs 0.75 [95\%CI 0.58- 0.98] respectively) although the benefit was not statistically significant in the MRI active subgroup, which again may be due to lack of power. The absolute gain of people with confirmed disability progression was $+10.5 \%$ using CDP-12 
(32.8\% in the ocrelizumab arm vs $43.3 \%$ in the placebo group), and $+7.8 \%$ using CDP-24 (30.7\% vs $38.5 \%$ respectively).

In France, at a first examination, the Transparency Committee concluded in April 2018 that ocrelizumab provided weak SMR and no added benefit (ASMR rating of V) in patients with PPMS matching the label indication [10]. This therefore contrasts with the claim by the Company (Roche) who submitted a request for moderate rating for added benefit (ASMR rating of III).

In July 2018, following re-examination by the Transparency Committee subsequent to an appeal by the Company, the SMR rating was revised from a weak to a moderate level yet the members voted unanimously that ASMR rating should remain unchanged at V [10]. This was the final decision made by the Transparency Committee.

Given the submitted evidence, relying on a clinical trial that only partially matched with the label indication, an ASMR rating of $\mathrm{V}$ for the label indication is unsurprising.

\begin{abstract}
Although there is no clinically relevant comparator for ocrelizumab in PPMS, the drug being granted a moderate SMR rating and an ASMR rating of $\mathrm{V}$ relative to placebo has meant ocrelizumab in PPMS falls outside the criteria for T2A list eligibility [25]. As a consequence, no pricing discussions appear to have been conducted between the French CEPS and the Company. Anecdotally, this means the cost-effectiveness opinion provided by the CEESP [32] with regards to PPMS became redundant for the purpose of decision-making.
\end{abstract}


As of September 2020, ocrelizumab for patients with PPMS matching the label indication is permissible in French hospitals [33] but is not reimbursed beyond Diagnosis Related Groups (DRG) tariffs, which means hospitals wishing to offer this option to patients have to fund the drug from their own budget. Conversely, ocrelizumab in RRMS patients is specifically refunded through T2A list [17]. This latter situation comes from the more favourable assessment by transparency committee for ocrelizumab in RRMS (ASMR rating in III in RRMS patients with early disease stage and signs of inflammatory disease)[34].

At NICE level, there were a number of points of discussion specific to the cost-effectiveness evaluation especially with regards to the inclusion of costs, disutilities applied for health states, the source of data to inform baseline transitions between EDSS states, or the possible waning of the drug's effect [16]. We have not attempted to compare the concerns raised by NICE and HAS on the inputs for the cost-effectiveness model because these elements were part of the decision making only in England and not in France.

In England, in a first (non-final) appraisal, NICE concluded in June 2018 that ocrelizumab did not represent good value for money compared with standard of care without ocrelizumab [12].

In subsequent submissions to NICE by the Company, updated results from the ORATORIO trial were presented, these especially deriving from the open-label extension phase of ORATORIO but the appraisal committee considered these results to be at high risk of performance bias. Based on the committee's preferred assumptions for the economic model, NICE retained its original stance of August 2018 that ocrelizumab did not represent good value for money within the NHS [16]. 
Finally, following further submission by the Company of a new commercially confidential arrangement regarding drug price, which were discussed in March 2019, NICE concluded that ocrelizumab was cost-effective for treating patients with early PPMS, resulting in a positive guidance finally issued in June 2019 [16].

\section{Expert Opinion}

In England ocrelizumab became available to patients within the NHS more than a year later than in France but on a permanent basis at a drug price that remains commercially confidential (figure 1). Conversely in France we have described how access to ocrelizumab occurred much early thanks to the ATU programme, but that it subsequently became unavailable to newly eligible patients because SMR and ASMR ratings precluded its registration on the T2A list.

As highlighted in a previous analysis of ixazomib for multiple myeloma [18], we found it paradoxical that a new drug is judged so promising that it is made available under the ATU programme, but it is eventually granted an ASMR rating of V based on the same set of data about clinical effectiveness.

The criteria used for determining ATU scheme eligibility and for ASMR rating are dissimilar; furthermore, these assessments are undertaken by different stakeholders, namely ANSM and HAS respectively. Irrespective of what maybe the most relevant decision to be taken, this paradoxical situation points to a requirement for a more consistent policy of access to innovation in France; current implementation allows temporal inequality among patients resulting in the real possibility of unfairness in access to treatments.

We have found that slight differences in submitted clinical effectiveness evidence (the ITT ORATORIO population only in France $v s$ the same + post-hoc defined MRI-active subgroup analyses in England), appear to have contributed to very different decisions in France and 
England; however it is evident that in England confidential pricing arrangements have been crucial whereas in France pricing discussion with the company has apparently not occurred within the scope of PPMS.

It is tempting to speculate that if post-hoc data used by NICE had been submitted to HAS the development of decision making in France may have followed a different pathway. Our view however is that given the post-hoc nature of these analyses subsequent stages in the appraisal would not have been different. Our opinion of no influence on outcome is supported by the absence of emphasis for the pre-specified subgroup analysis in patients with $\mathrm{T} 1 \mathrm{Gd}+$ at inclusion since their time to CDP was not significantly delayed.

In England, had the Company presented a submission based on the ITT ORATORIO population, and not the MRI-active subgroup, we may speculate that in the absence of a very favourable pricing arrangement NICE would have issued a final guidance not recommending ocrelizumab for PPMS. For the most favourable scenario, that is where the data considered closely matched the label indication, it took more than a year and three appraisal committee meetings to arrive at a final positive decision.

Regardless of the impact of different contents of submission for reimbursement, it would make sense that Companies in general share the same material regarding the clinical effectiveness and safety of new technologies across HTA bodies particularly within Europe.

This case-study exemplifies the greater emphasis put on clinical effectiveness in France to support positive decisions on reimbursement. Indeed, HAS use ASMR rating as a core element of decision making in France, as illustrated by criteria for T2A list eligibility. 
As previously emphasised, NICE use cost-effectiveness evaluation as a key element of decision making which means NICE is likely to recommend any therapy provided the ICER falls below $£ 30,000 / \mathrm{QALY}$.

Both French and English paradigms of decision-making although applied in an imperfect world and sometimes tortuous and prolonged in practice, in general appear fit for purpose. It is important in our view that each approach is applied consistently to all drugs and more broadly to all health technologies.

While we have emphasised the logical nature of $T 2 A$ list eligibility criteria in France, a major limitation is that by definition these apply only to drugs with potential refund in addition to DRGs tariffs, which most of the time only pertains to intravenously administered drugs.

For drugs outside this scope, such as those administered orally and used in ambulatory care, benchmark to determine eligibility to reimbursement is not based on ASMR rating, but on SMR. Ambulatory care drugs with sufficient SMR and ASMR rating of V are reimbursed, if the pharmaceutical company accepts to negotiate with CEPS a price that is lower than those of the comparators, or the standard of care, if there is no comparator on the French drug market [18]. Had ocrelizumab been an orally administered drug, as opposed to an intravenously given therapy, we speculate that the fate of reimbursement in France might have differed.

One may argue that this opinion is based on a single observation hence lack generalisability, however we would point out that in the case that a drug is granted with ASMR rating of V, it has almost no chance to obtain a dedicated reimbursement if it requires IV infusion while it may have some chance in the event the drug is an oral form and used in ambulatory care. 
This present work has some limitations. Due to our method of analysis we did not include interviews with stakeholders; we were unable to access documents excluded from the public domain and consequently we may have not been able to fully capture the full complexity of the decision-making process. Also, we have only focussed on a single case-study which means our observations may not be widely applicable to all health technologies appraisals.

Lastly, we have not undertaken a comprehensive analysis of HTA across all European countries. Given the apparent disparate market access for ocrelizumab in PPMS, such analysis would be worthwhile but complex and necessitate overcoming difficulties of multiplicity of languages.

In conclusion, based on our specific observation of England and France, we have emphasised that differences on the HTA process and criteria for reimbursement can result in differences in timing and outcome for the access to market of newly licensed drugs. Future research work based on semi-directed interviews of stakeholders could be worth considering to complete our analysis.

\section{Funding}

This paper is not funded.

\section{Declaration of Interests}

The authors have no relevant affiliations or financial involvement with any organization or entity with a financial interest in or financial conflict with the subject matter or materials discussed in the manuscript. This includes employment, consultancies, honoraria, stock ownership or options, expert testimony, grants or patents received or pending, or royalties.

\section{Reviewer Disclosures}

Peer reviewers on this manuscript have no relevant financial relationships or otherwise to disclose. 


\section{References}

1. Montalban X, Hauser SL, Kappos L, Arnold DL, Bar-Or A, Comi G, de Seze J, Giovannoni G, Hartung H-P, Hemmer B, Lublin F, Rammohan KW, Selmaj K, Traboulsee A, Sauter A, Masterman D, Fontoura P, Belachew S, Garren H, Mairon N, Chin P, Wolinsky JS, ORATORIO Clinical Investigators. Ocrelizumab versus Placebo in Primary Progressive Multiple Sclerosis. N Engl J Med. 2017;376:209-20.

2. Hauser SL, Bar-Or A, Comi G, Giovannoni G, Hartung H-P, Hemmer B, Lublin F, Montalban X, Rammohan KW, Selmaj K, Traboulsee A, Wolinsky JS, Arnold DL, Klingelschmitt G, Masterman D, Fontoura P, Belachew S, Chin P, Mairon N, Garren H, Kappos L. Ocrelizumab versus Interferon Beta-1a in Relapsing Multiple Sclerosis. N Engl J Med. 2017;376:221-34.

3. Anonymous. Ocrevus. European Medicines Agency. 2018. https://www.ema.europa.eu/en/medicines/human/EPAR/ocrevus (accessed 10 July 2020).

4. Armoiry X, Kan A, Melendez-Torres GJ, Court R, Sutcliffe P, Auguste P, Madan J, Counsell C, Clarke A. Short- and long-term clinical outcomes of use of beta-interferon or glatiramer acetate for people with clinically isolated syndrome: a systematic review of randomised controlled trials and network meta-analysis. J Neurol. 2018;265:999-1009.

5. Melendez-Torres GJ, Armoiry X, Court R, Patterson J, Kan A, Auguste P, Madan J, Counsell C, Ciccarelli O, Clarke A. Comparative effectiveness of beta-interferons and glatiramer acetate for relapsing-remitting multiple sclerosis: systematic review and network meta-analysis of trials including recommended dosages. BMC Neurol. 2018;18:162.

6. He D, Zhang C, Zhao X, Zhang Y, Dai Q, Li Y, Chu L. Teriflunomide for multiple sclerosis. Cochrane Database of Systematic Reviews. 2016.

7. Zhang J, Shi S, Zhang Y, Luo J, Xiao Y, Meng L, Yang X. alemtuzumab versus interferon beta 1a for relapsing-remitting multiple sclerosis. Cochrane Database of Systematic Reviews. 2017.

8. Melendez-Torres G, Auguste P, Armoiry X, Maheswaran H, Court R, Madan J, Kan A, Lin S, Counsell C, Patterson J, Rodrigues J, Ciccarelli O, Fraser H, Clarke A. Clinical effectiveness and cost-effectiveness of beta-interferon and glatiramer acetate for treating multiple sclerosis: systematic review and economic evaluation. Health Technol Assess. 2017;21:1-352.

9. Airas L, Nylund M, Mannonen I, Matilainen M, Sucksdorff M, Rissanen E. Rituximab in the treatment of multiple sclerosis in the Hospital District of Southwest Finland. Multiple Sclerosis and Related Disorders. 2020;40:101980.

10. OCREVUS (ocrelizumab), immunosuppresseur (SEP d'emblée progressive). Haute Autorité de Santé. n.d. https://www.has-sante.fr/jcms/c_2863557/fr/ocrevus-ocrelizumabimmunosuppresseur-sep-d-emblee-progressive (accessed 10 July 2020).

11. Achtnichtsa L, Chanb A, Czaplinskic A, Derfussd T, Du Pasquiere R, Findlinga O, et al. Particularités de l'immunothérapie de la sclérose en plaques en Suisse, Swiss Medical Forum, Forum Médical Suisse, 2019;19(41-42):676-685 n.d.

12. Documents | Ocrelizumab for treating primary progressive multiple sclerosis | Guidance | NICE n.d. https://www.nice.org.uk/guidance/ta585/history (accessed 10 July 2020).

13. ocrelizumab (Ocrevus). Scottish Medicine Consortium. n.d. https://www.scottishmedicines.org.uk/medicines-advice/ocrelizumab-ocrevus-fullsmc2223/ (accessed 15 July 2020).

14. Gemeinsamer Bundesausschuss. Tragende Gründe: zum Beschluss des Gemeinsamen Bundesausschusses über eine Änderung der Arzneimittel-Richtlinie (AM-RL): Anlage 
XII - Beschlüsse über die Nutzenbewertung von Arzneimitteln mit neuen Wirkstoffen nach § 35a SGB V - Ocrelizumab 2018.

15. Adler AI, Knight H. Ocrelizumab for primary progressive multiple sclerosis. The Lancet Neurology. 2019;18:816-7.

16. Auguste P, Colquitt J, Connock M, Loveman E, Court R, Ciccarelli O, Counsell C, Armoiry X. Ocrelizumab for Treating Patients with Primary Progressive Multiple Sclerosis: An Evidence Review Group Perspective of a NICE Single Technology Appraisal. PharmacoEconomics. 2020;38:527-36.

17. Arrêté du 22 février 2019 modifiant la liste des spécialités pharmaceutiques prises en charge en sus des prestations d'hospitalisation mentionnée à l'article L. 162-22-7 du code de la sécurité sociale $\mid$ Legifrance n.d.

https://www.legifrance.gouv.fr/eli/arrete/2019/2/22/SSAS1825280A/jo/texte (accessed 10 July 2020).

18. Armoiry X, Spath H-M, Clarke A, Connock M, Sutcliffe P, Dussart C. Comparison of health technology assessment for new medicines in France and England: an example based on ixazomib for patients with relapsed or refractory multiple myeloma. Journal of Market Access \& Health Policy. 2019;7:1648971.

19. National Institute for Health and Care Excellence. Overview of technology appraisals for patient and carer organisations, Public Involvement Programme, April 2014 n.d.

20. McCabe C, Claxton K, Culyer AJ. The NICE cost-effectiveness threshold: what it is and what that means. Pharmacoeconomics. 2008;26:733-44.

21. Topic selection | Our programmes | What we do | About. NICE. n.d. https://www.nice.org.uk/about/what-we-do/our-programmes/topic-selection (accessed 11 September 2020).

22. Haute Autorité de Santé - Le service médical rendu (SMR) et l'amélioration du service médical rendu (ASMR) n.d. https://www.has-sante.fr/portail/jcms/r_1506267/fr/leservice-medical-rendu-smr-et-l-amelioration-du-service-medical-rendu-asmr (accessed 2 July 2019).

23. Haute Autorité de Santé (HAS). Évaluation des médicaments- Doctrine de la commission de la transparence: Principes d'évaluation de la CT relatifs aux médicaments en vue de leur accès au remboursement 2018.

24. Giorgi D. [The French medecine pricing committee]. Ann Pharm Fr. 2017;75:373-84.

25. Décret $\mathrm{n}^{\circ}$ 2016-349 du 24 mars 2016 relatif à la procédure et aux conditions d'inscription des spécialités pharmaceutiques sur la liste mentionnée à l'article L. 162-22-7 du code de la sécurité sociale. 2016.

26. Toumi M, Motrunich A, Millier A, Rémuzat C, Chouaid C, Falissard B, Aballéa S. Analysis of health economics assessment reports for pharmaceuticals in France understanding the underlying philosophy of CEESP assessment. J Mark Access Health Policy. 2017;5:1344088.

27. Haute Autorité de Santé (HAS) - Décision n² 2018.0022/DC/SEESP du 21 février 2018 du collège de la Haute Autorité de santé constatant l'impact significatif du produit OCREVUS ${ }^{\circledR} 300 \mathrm{mg}$ sur les dépenses de l'assurance maladie n.d.

28. Haute Autorité de Santé. L'évaluation médico-économique des Médicaments et Dispositifs Médicaux, Dossier de presse, Conférence de presse, 18 décembre 2014 n.d.

29. Qu'est ce qu'une autorisation temporaire d'utilisation? - ANSM : Agence nationale de sécurité du médicament et des produits de santé n.d. https://www.ansm.sante.fr/Activites/Autorisations-temporaires-d-utilisation-ATU/Qu-estce-qu-une-autorisation-temporaire-d-utilisation/(offset)/0 (accessed 3 January 2019).

30. Mays N, Pope C. Qualitative research: Observational methods in health care settings. BMJ. 1995;311:182-4. 
31. OCRELIZUMAB $300 \mathrm{mg}$, solution à diluer pour perfusion - ANSM : Agence nationale de sécurité du médicament et des produits de santé n.d.

https://www.ansm.sante.fr/Activites/Autorisations-temporaires-d-utilisation-ATU/ATUarretees/Liste-des-ATU-arretees/OCRELIZUMAB-300-mg-solution-a-diluer-pourperfusion (accessed 10 July 2020).

32. Haute Autorité de Santé (HAS). Ocrevus ${ }^{\circledR}$ (ocrelizumab): avis d'efficience Analyse d'impact budgétaire dans la SEP 2018.

33. Arrêté du 25 février 2019 modifiant la liste des spécialités pharmaceutiques agréées à l'usage des collectivités et divers services publics. n.d.

34. OCREVUS (SEP récurrente). Haute Autorité de Santé. n.d. https://www.hassante.fr/jcms/c_2868189/fr/ocrevus-sep-recurrente (accessed 15 July 2020). 


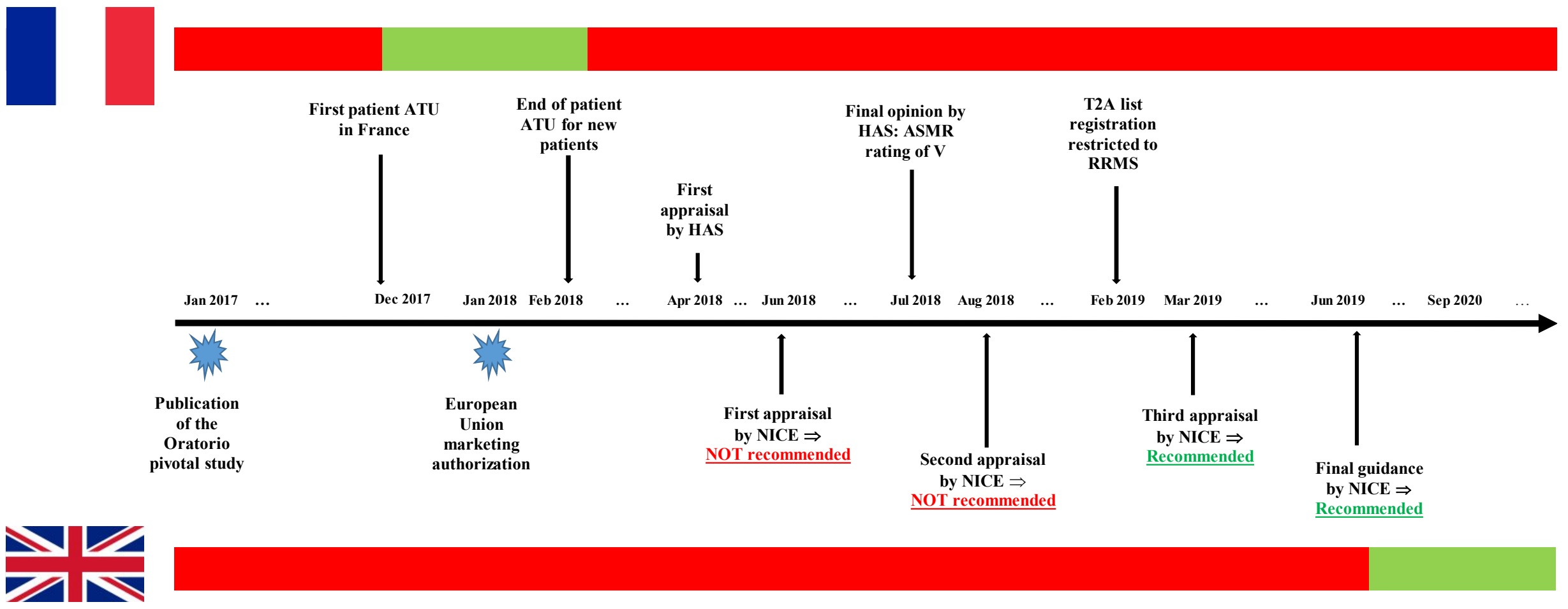

\section{Figure 1: Comparative availability over time of ocrelizumab for new patients with PPMS between France and England and main}

\section{milestones of the appraisal}

The red parts denote periods of time during which ocrelizumab was not reimbursed by each country health care system, the green parts denote periods of time during which ocrelizumab was reimbursed by each country health care system, NICE = National Institute for Health and Care Excellence; HAS= French High Health Authority; ASMR = improvement of actual medical benefit; PPMS= primary progressive multiple sclerosis; ATU= Early Access Program, RRMS= relapsing remitting MS 